\title{
Proceedings of the 5th plenary meeting of the COST E21 Action
}

\author{
Thessaloniki (Greece)
}

27-29th November 2003

\begin{abstract}
The Cost E21 Action entitled "The role of forest for mitigating greenhouse gas emissions" started in December 1999 and ended in June 2004. The main objective of the Action was to bring together scientists of common interest to consider and progress the contribution of forests and forestry to the mitigation of greenhouse effects. The Action has addressed the complex issue of carbon accounting with the objective of contributing to the development of an agreed carbon accounting strategy for European forests within the framework of the Kyoto protocol. The Action has further seeked to define and quantify the influence of forest management on the carbon balance of forests, investigating both the biogeochemical and socio-economic impacts of management practices and exploring the potential for enhancing the role of forests in the mitigation of greenhouse effects. A series of papers appeared in Biotechnology, Agronomy, Society and Environment, and introduced by Laitat et al. ${ }^{1}$, provided the state-of-the-art in the forest sector carbon reporting for participating countries and helped realise how acute was the need to harmonise and coordinate carbon inventory methods among European countries. Progress was realised then owing to the active information exchange allowed by short-term scientific missions, plenary and subgroups meeting and also through the European Research Project CARBOINVENT which emerged from the Action. Several participants of the COST E21 Action were also active in the preparation of the Good Practice Guidance for Land Use, Land Use Change and Foresrry appeared in 2004.

The fifth plenary meeting of the COST E21 Action was hosted by Dr Radoglou (NAGREF) in Thessaloniki (Greece) 27-29th November 2003, where 22 oral communications and 36 posters were presented with the aim of synthetising and updating results of ongoing national research on the carbon cycle in forest. The following eight articles present original contributions covering various aspects of the topics addressed during the meeting, from process studies to regional modelling, and reflecting the diversity of approaches and spatial scales considered in carbon studies.

Indeed, some quite significant progress have been made since 1990 in the understanding of the environmental, ecophysiological and silvicultural controls of the forest carbon cycle, and new tools for assessing the present and future impacts of forest on greenhouse gas concentrations have been proposed. We think the COST E21 Action played a part in this global progress and was particularly successful bringing together scientists from 23 European countries for exchanging information, harmonising their approaches and undertake new research projects and actions at the European level.
\end{abstract}

On behalf of the organising committee:

Loustau Denis (Working Group1 coordinator), Unité EPHYSE, INRA-Bordeaux, BP 81, 33883 Villenave d'Ornon Cedex, France.

Radoglou Kallioppi

(Scientific organiser),

National Agricultural Research Foundation,

Vassilika,

57006 Thessaloniki,

Greece.

Lindner Marcus (Working Group2 coordinator), Forest Ecology and Management,

European Forest Institute,

Torikatu 34,

80100 Joensuu,

Finland.
Laitat Eric (COST E21 Action Chairman), Faculté des Sciences Agronomiques,

Passage des Déportés 2,

Gembloux, 5030 Belgium.

Linder Sune,

Swedish University of Agricultural Sciences, Southern Swedish Forest Research Centre, PO Box 49,

23053 ALNARP, Sweden.

Karjalainen Timo

Finnish Forest Research Institute,

Joensuu Research Centre,

PO Box 68,

80101 Joensuu, Finland.

\footnotetext{
${ }^{1}$ Laitat E., Karjalainen T., Loustau D., Lindner M., Introduction: Towards an integrated scientific approach for carbon accounting in forest ecosystems, Bioechnol. Agron. Soc. Environ. 4 (2000) 241-251.
} 